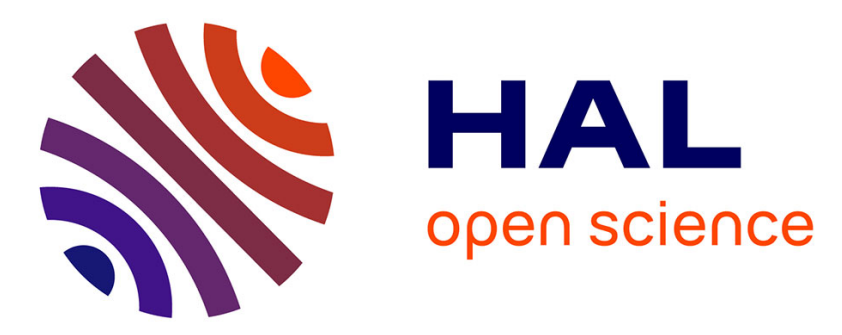

\title{
Le phénomène de la continuité auditive et la répartition de la sonie entre flux auditifs
}

\author{
S. Mcadams, M.-C. Botte, C. Drake
}

\section{To cite this version:}

S. Mcadams, M.-C. Botte, C. Drake. Le phénomène de la continuité auditive et la répartition de la sonie entre flux auditifs. Journal de Physique IV Proceedings, 1994, 04 (C5), pp.C5-383-C5-386. 10.1051/jp4:1994579 . jpa-00253074

\section{HAL Id: jpa-00253074 https://hal.science/jpa-00253074}

Submitted on 1 Jan 1994

HAL is a multi-disciplinary open access archive for the deposit and dissemination of scientific research documents, whether they are published or not. The documents may come from teaching and research institutions in France or abroad, or from public or private research centers.
L'archive ouverte pluridisciplinaire HAL, est destinée au dépôt et à la diffusion de documents scientifiques de niveau recherche, publiés ou non, émanant des établissements d'enseignement et de recherche français ou étrangers, des laboratoires publics ou privés. 


\title{
Le phénomène de la continuité auditive et la répartition de la sonie entre flux auditifs
}

\author{
S. McADAMS ${ }^{* * *}$, M.-C. BOTTE ${ }^{*}$ et C. DRAKE* \\ * Laboratoire de Psychologie Expérimentale, CNRS, Université René Descartes, 28 rue Serpente, \\ 75006 Paris, France \\ ${ }^{* *}$ IRCAM, 1 place Stravinsky, 75004 Paris, France
}

\begin{abstract}
A pure tone whose amplitude alternates between two levels can give rise to a perception not of alternation, but of an intermittent sound superimposed on a continuous sound. The more intense part is segregated into two simultaneous parts, one assigned to the continuous sound and the other to the intermittent sound. According to the theory of auditory scene analysis, the loudness of the intermittent part is computed from what is left over after having subtracted the energy assigned to the continuous part. To test this hypothesis, we presented subjects with sequences composed of a $1 \mathrm{kHz}$ pure tone alternating between two levels every $200 \mathrm{~ms}$. The difference in levels was 2,6 or $10 \mathrm{~dB}$, the lower one roving randomly around $60 \mathrm{~dB}$. Subjects were asked to adjust a continuous or intermittent comparison sequence to have the same loudness as the corresponding part of the alternating sequence. The theory predicts zero difference between the lower level and the adjusted continuous sound, but differences of $-3.3,-6.0$ and $-13.7 \mathrm{~dB}$ between the higher level segments and the adjusted intermittent sound. Results conform qualitatively to the theory though the adjusted level of the intermittent sound is higher than expected. Adjustments errors can be partly explained by errors in partitioning the stimulus energy and partly by the weakness of the phenomenon at small level differences.
\end{abstract}

\section{INTRODUCTION}

Afin de réagir de façon appropriée à un environnement sonore, un organisme doit pouvoir séparer les informations sensorielles provenant de sources distinctes et regrouper les informations provenant d'une même source qui sont distribuées à travers les canaux fréquentiels et dans le temps. Ce problème est abordé par la théorie de l'analyse des scènes auditives [1]. Warren et coll. [2] ont montré qu'un son de niveau sonore relativement faible qui alterne avec un son de niveau plus élevé est perçu comme continuant "derrière" le son fort, si le contenu fréquentiel du son fort recouvre celui du son faible (Fig. 1). Ce phénomène s'appelle la "continuité auditive". Un des mécanismes de traitement primaire proposé pour contribuer à cette organisation des informations auditives est la stratégie «ancien-plus-nouveau» selon Bregman [1]. Cette stratégie effectue une sorte d'interpolation entre les occurrences du son faible avant et après son interruption par le son fort (restauration perceptive), à condition que les informations auditives indiquent que le son faible aurait pu être présent durant les occurrences du son fort. Le laps de temps occupé par le son fort est alors interprété comme résultant du mélange intermittent du son faible (ancien) et du son fort (nouveau). En outre, les attributs auditifs du son fort sont calculés après soustraction des informations censées faire partie du son faible. Warren et coll. [3] ont montré qu'un șon sinusoïdal de niveau faible interrompu périodiquement par un son sinusoïdal de fréquence et de phase identiques mais de niveau plus élevé, donne lieu à la perception d'un son continu de sonie faible et d'une séquence intermittente dont la sonie dépend de la différence de niveau (Fig. 1). Si ce mécanisme est capable de restituer une représentation véridique du monde sonore selon la stratégie "ancien-plus-nouveau", la sonie de la séquence intermittente devrait être calculable à partir de la différence entre l'amplitude globale de la partie forte et l'amplitude de la partie faible, cette dernière quantité étant affectée au son continu.

Afin de tester cette hypothèse, nous avons mené une expérience (Exp. 3) dans laquelle trois différences de niveau $(\Delta N)$ sont présentées dans des séquences de niveaux alternants (Fig. 1). Selon les prédictions de la théorie, pour les différences de $+2,+6$ et $+10 \mathrm{~dB}$ du niveau fort par rapport à un niveau faible de $60 \mathrm{~dB}$ SPL, un auditeur essayant d'apparier le niveau d'une séquence de comparaison à la partie intermittente de la 
séquence alternante, devrait l'ajuster respectivement à $-13.7,-6.0$ et $-3.3 \mathrm{~dB}$ par rapport au niveau physique de la partie forte. On remarquera que dans le cas d'une différence de $2 \mathrm{~dB}$. le niveau de la séquence intermittente devrait être plus faible $(48.3 \mathrm{~dB})$ que celui du son continu $(60 \mathrm{~dB})$. Si l'organisation en flux n'affecte pas la sonie perçue, nous devrions obtenir des ajustements conformes aux niveaux physiques présentés. Le niveau apparié à la partie continue devrait être identique au niveau physique dans les deux cas. Nous avons également mené deux expériences contrôle (Exp. 1 et 2) pour vérifier la précision des ajustements de niveau et pour entraîner les sujets.
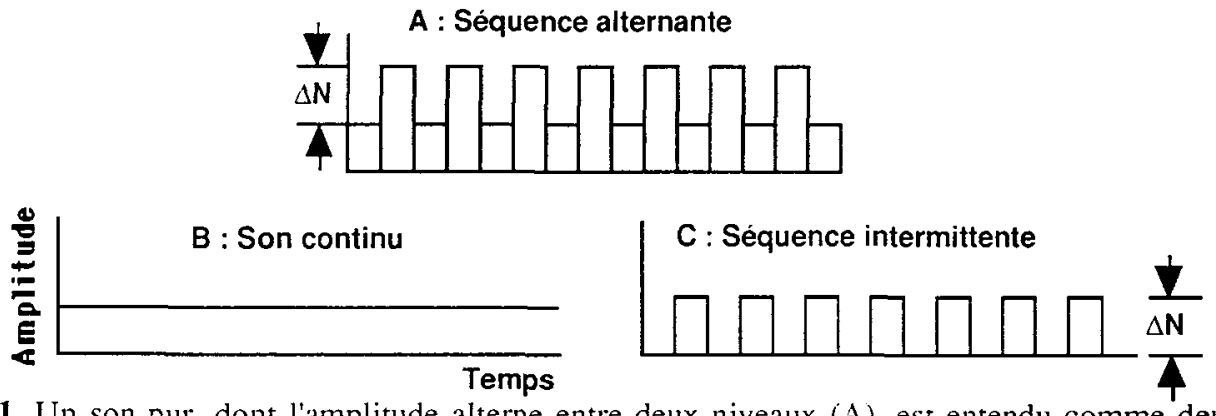

Figure 1. Un son pur, dont l'amplitude alterne entre deux niveaux (A), est entendu comme deux flux auditifs superposés : un son continu (B) et une séquence intermittente (C). L'hypothèse testée pose que la sonie de la séquence intermittente est équivalente à celle d'un son de niveau $\Delta N$.

\section{EXPERIENCE 1 : Séquences alternantes présentées de façon dichotique.}

Le but de cette expérience contrôle est de vérifier la précision de l'ajustement du niveau avec un stimulus de même configuration temporelle que celui de l'expérience 3 mais ne procurant pas une perception de continuité.

\subsection{Méthode}

Lors de chaque essai, on présente en alternance un stimulus de référence et un stimulus de niveau réglable par le sujet à l'aide d'un potentiomètre, jusqu'à ce que l'ajustement soit terminé. Le stimulus de référence consiste en une séquence alternante de 15 sons sinusoïdaux de $1 \mathrm{kHz}$ et d'une durée de $200 \mathrm{~ms}$ dont l'amplitude alterne entre un niveau fort et un niveau faible (Fig. 1, A). Le niveau faible est choisi au hasard dans l'ensemble $60 \pm\{1,3,5\} \mathrm{dB}$ SPL afin d'éviter une mémorisation de la sonie du niveau faible. Le niveau fort varie de façon systématique entre trois valeurs : $+2,+6$ et $+10 \mathrm{~dB}$ par rapport au niveau faible. Les sons faibles sont toujours présentés à l'oreille gauche et les sons forts à l'oreille droite. Les rampes linéaires des attaques et extinctions durent $5 \mathrm{~ms}$ et se croisent à $2.5 \mathrm{~ms}$. La phase de la forme d'onde est identique dans les deux oreilles. Au début de l'expérience, nous présentons les trois types de séquences de référence au sujet en précisant que le niveau sera plus intense dans l'oreille droite et en lui demandant de décrire ce qu'il entend. Aucun sujet n'a rapporté une sensation de continuité.

Le stimulus à ajuster consiste en une séquence de 7 sons de $1 \mathrm{kHz}$ et d'une durée de $200 \mathrm{~ms}$ séparés par des silences de $200 \mathrm{~ms}$. La pente linéaire des attaques et extinctions dure $5 \mathrm{~ms}$. La séquence est présentée soit à l'oreille gauche, soit à l'oreille droite. Le niveau de cette séquence au début d'un essai a été choisi au hasard dans l'ensemble $\pm\{7,8,9,10\} \mathrm{dB}$ par rapport au niveau faible. On demande au sujet d'ajuster son niveau pour qu'il soit identique à celui de la partie de la séquence de référence entendue dans la même oreille. L'expérience comporte donc 6 conditions expérimentales (présentation de la séquence d'ajustement à droite ou à gauche pour chacune des 3 différences de niveau). Chaque sujet a passé 5 blocs de ces 6 conditions présentées dans un ordre aléatoire au sein d'un bloc donné. Huit sujets (y compris les trois auteurs) ont participé à l'expérience.

\subsection{Résultats}

La variable dépendante est la différence entre le niveau physique et le niveau ajusté. Les erreurs moyennes varient de -0.1 à $1.3 \mathrm{~dB}$ indiquant une tendance à surestimer le niveau référence (Fig. 2). La précision de l'ajustement est meilleure pour l'oreille droite lorsqu'on doit égaler le niveau le plus fort et pour l'oreille gauche lorsque la différence de niveau interaurale est la plus faible. Une analyse de variance avec mesures répétées a été effectuée pour 3 variables indépendantes : différence de niveau interaurale (3), oreille de présentation de la séquence d'ajustement (2), répétition (5). Seule l'interaction entre différence de niveau et oreille est significative, indiquant une différence de précision d'ajustement entre les deux oreilles pour les conditions $+2 \mathrm{~dB}(\mathrm{OD} 1.3 \mathrm{~dB}$; OG $0.3 \mathrm{~dB}$ ) et $+10 \mathrm{~dB}$ (OD $-0.1 \mathrm{~dB} ;$ OG $0.9 \mathrm{~dB}$ ). Les deux seules conditions pour lesquelles le niveau ajusté est différent du niveau physique sont pour l'oreille droite à $+2 \mathrm{~dB}$ et pour 
l'oreille gauche à $+10 \mathrm{~dB}$. Nous pouvons conclure de cette étude, que les erreurs d'ajustement en l'absence de perception de continuité, bien que significatives, sont reiativement petites $(<1.5 \mathrm{~dB}$ en moyenne).

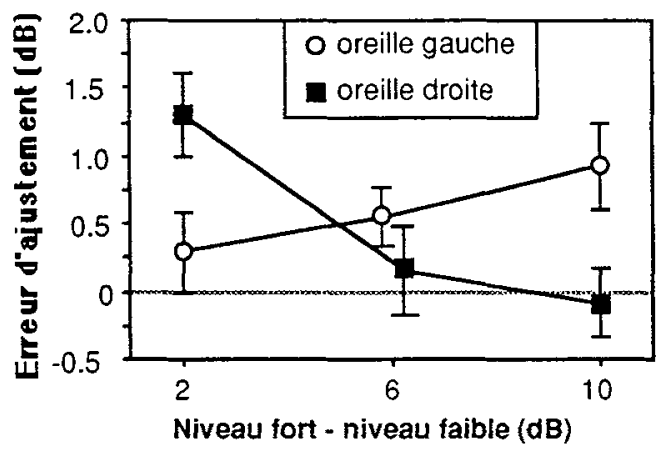

Figure 2. Expérience 1 : Erreur d'ajustement du niveau en fonction de la différence interaurale de niveau dans la séquence de référence. Barres verticales $= \pm 1$ erreur standard.

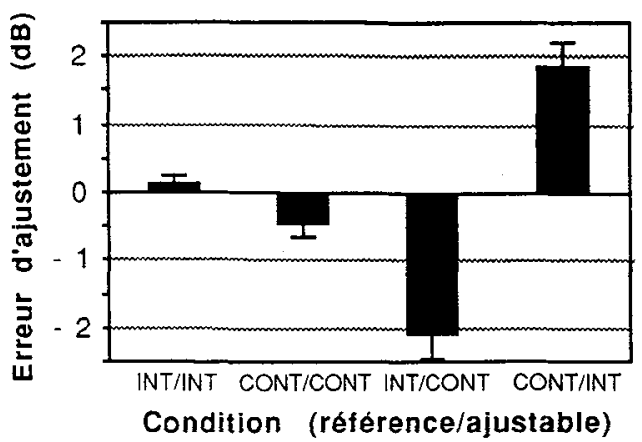

Figure 3. Expérience 2 : Erreur d'ajustement du niveau pour les comparaisons de stimuli continus (CONT) et intermittents (INT). Barres verticales = 1 erreur standard.

\section{EXPERIENCE 2 : Comparaison de sons intermittents et continus.}

Le but de cette deuxième expérience contrôle était de vérifier si la précision d'ajustement dépend de la continuité du stimulus de référence ou du stimulus ajustable.

\subsection{Méthode}

Deux types de stimuli ont été utilisé : 1) une séquence intermittente (INT) de 7 sons de $1 \mathrm{kHz}$ et d'une durée de $200 \mathrm{~ms}$ séparés par des silences de $200 \mathrm{~ms}$ ayant des rampes linéaires de $5 \mathrm{~ms} ; 2$ ) un son continu (CONT) de $1 \mathrm{kHz}$ d'une durée de $2600 \mathrm{~ms}$. Chaque type a été présenté soit comme stimulus de référence, soit comme stimulus ajustable, ce qui donne 4 comparaisons : INT/INT, CONT/CONT, INT/CONT, CONT/INT. Le niveau du stimulus de référence et le niveau de départ du stimulus ajustable ont été choisis comme dans l'expérience 1. Les stimuli ont été présentés de façon binaurale (diotique). La tâche du sujet était d'apparier la sonie des deux stimuli en ajustant le niveau du stimulus de comparaison. Chacun des 8 sujets a passé 5 blocs de ces 4 conditions présentées dans un ordre aléatoire au sein d'un bloc.

\subsection{Résultats}

La variable dépendante est la différence entre le niveau physique et le niveau ajusté. Les erreurs moyennes varient de -2.2 à $1.8 \mathrm{~dB}$ (Fig. 3). La précision d'ajustement est très bonne quand les deux stimuli sont de même type. Lorsqu'ils sont de types différents, l'erreur est d'environ $2 \mathrm{~dB}$. Le fait que les erreurs pour INT/CONT et CONT/INT soient de signes opposés suggère une tendance à sous-estimer le niveau du stimulus intermittent par rapport au stimulus continu. Une analyse de variance avec mesures répétées a été effectuée pour 2 variables indépendantes : condition de comparaison (4) et répétition (5). Seul l'effet principal de condition est significatif. Deux conditions ont une implication directe dans l'expérience principale (Exp. 3) : INT/INT et CONT/CONT. Pour la première, le niveau ajusté n'est pas significativement différent du niveau physique. Le niveau ajusté de la seconde est significativement inférieur au niveau physique, mais la différence n'est que $0.5 \mathrm{~dB}$. Nous pouvons conclure que les sujets ont une précision de l'ordre de $0.5 \mathrm{~dB}$ dans ce genre de tâche en l'absence de variations de niveau dans le stimulus de référence.

\section{EXPERIENCE 3 : Séquences diotiques procurant une sensation de continuité auditive.}

Le but de cette expérience était de tester l'hypothèse selon laquelle l'amplitude d'un signal de niveau alternant organisé en deux flux distincts (continu et intermittent) est partitionnée en deux quantités : l'une correspondant à celle affectée au flux continu, et l'autre correspondant à ce qui reste ( $\Delta \mathrm{N}$ dans la Fig. 1$)$, affectée au flux intermittent.

\subsection{Méthode}

Lors de chaque essai, un stimulus de référence était présenté en alternance avec un stimulus ajustable. Le stimulus de référence consistait en un son sinusoïdal de $1 \mathrm{kHz}$ dont l'amplitude variait toutes les $200 \mathrm{~ms}$ entre un niveau faible, choisi au hasard parmi $60 \pm\{1,3,5\} \mathrm{dB}$, et un niveau fort qui était, selon la condition, 2,6 ou $10 \mathrm{~dB}$ au-dessus du niveau faible. Les pentes de changement linéaire d'amplitude était de $5 \mathrm{~ms}$. Pendant une phase de familiarisation, ces stimuli ont été présentés aux sujets qui devaient décrire ce qu'ils entendaient. Tous les sujets ont rapporté une perception double composée d'un son intermittent superposé à un son continu, bien que cette sensation soit moins nette pour une différence de $2 \mathrm{~dB}$. 
Dans la phase test, ce stimulus de référence a été comparé à un stimulus ajustable de $1 \mathrm{kHz}$ qui était soit continu, soit intermittent. Le niveau de départ a été choisi au hasard (comme dans l'expérience 1). Si le stimulus ajustable était continu, le sujet devait ajuster son niveau pour apparier sa sonie à celle de la partie du stimulus de référence qui lui paraissait continue. Si le stimulus ajustable était intermittent, le sujet devait apparier sa sonie à celle de la partie intermittente du stimulus de référence. Six conditions expérimentales (3 différences de niveau. 2 types de stimuli ajustables) on été présentées dans un ordre aléatoire dans chaque bloc d'essais. Chacun des 8 sujets a passé 5 blocs.

\subsection{Résultats}

La variable dépendante est la différence entre le niveau physique et le niveau ajusté. Des tests $t$ de Student bilatéraux ont été effectués. L'erreur n'est pas significativement différente de zéro pour les stimuli ajustables continus (Fig. 4). Pour les séquences ajustables intermittentes le niveau ajusté est significativement inférieur au niveau physique pour tous les degrés de différence. Comme le prédit la théorie, la différence entre le niveau ajusté et le niveau physique est grande pour un petit $\Delta \mathrm{N}$ et décrô̂t progressivement avec l'augmentation de $\Delta \mathrm{N}$. Cependant, les niveaux ajustés sont significativement supérieurs aux valeurs théoriques pour $\Delta \mathrm{N}$ de +2 et $+6 \mathrm{~dB}$. En outre, la variabilité des niveaux ajustés est plus grande pour $\mathrm{J}=+2 \mathrm{~dB}$, ce qui témoigne de la difficulté à percevoir le phénomène avec ce stimulus.

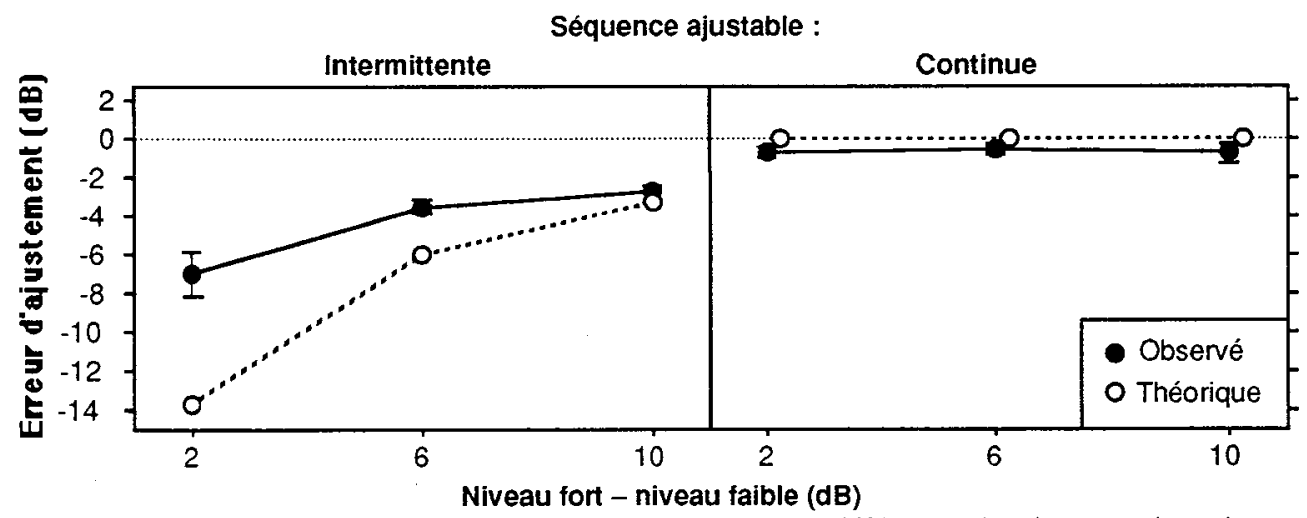

Figure 4. Expérience 3: Erreur d'ajustement en fonction de la différence de niveau présentée par une séquence référence de niveau alternant. Barres verticales $= \pm 1$ erreur standard.

\section{DISCUSSION}

Les deux premières expériences montrent que les sujets sont relativement précis dans leurs ajustements de niveau lorsqu'on leur demande d'apparier la sonie des stimuli ajustables, continus ou intermittents, avec celle de stimuli de référence semblables. L'erreur moyenne est de l'ordre de 0.1 à $1.3 \mathrm{~dB}$. Confrontés à des séquences de niveau alternant qui donnent la sensation d'un son intermittent superposé à un son continu, les sujets ajustent un son continu à un niveau pratiquement identique au niveau faible, mais n'ajustent jamais un son intermittent au niveau fort. Dans ce dernier cas, les ajustements sont systématiquement inférieurs au niveau physique et d'autant plus inférieurs que la différence de niveau au sein de la référence est petite. Le fait que ces niveaux sont toutefois supérieurs à ceux prédits par la théorie de l'analyse des scènes auditives suggère plusieurs interprétations : soit les sujets surestiment le niveau du son intermittent dans une séquence alternante, allant dans le sens du niveau du son continu qui est toujours présent, soit ils ne font pas une répartition de l'énergie du signal selon le mécanisme proposé par la théorie. Il existe apparemment une sousestimation du niveau continu de 0.5 à $0.7 \mathrm{~dB}$ et une surestimation du niveau intermittent allant de 0.6 à 6.7 $\mathrm{dB}$. Mais on peut reconstruire les niveaux forts "perçus" en recombinant les niveaux ajustés dans l'expérience pour les sons continus et pour les sons intermittents, puis comparer ces valeurs aux niveaux réels présentés. Les niveaux réels nominaux était 62,66 et $70 \mathrm{~dB}$. Les niveaux reconstruits sont $63.7,66.9$ et $70.3 \mathrm{~dB}$; les erreurs globales sont donc respectivement de $+1.7,+0.9$ et $+0.3 \mathrm{~dB}$, valeurs proches des erreurs d'ajustement pour la séquence forte (oreille droite) de l'expérience 1. L'erreur pour la condition +2 $\mathrm{dB}$ reflète également le manque de netteté du phénomène perçu par les sujets pour des petites différences de niveau. Nous concluons que le mécanisme théorique proposé n'est pas loin de la vérité compte tenu de l'influence de la méthode de mesure sur le phénomène étudié.

\section{REFERENCES}

[1] Bregman, A.S. Auditory scene analysis, Chap. 3. (MIT Press, Cambridge, MA, 1990).

[2] Warren, R.M. Auditory perception: A new synthesis, Chap. 6. (Pergamon Press, New York, 1982).

[3] Warren, R.M., Obusek, C.J. \& Ackroff, J.M. Science 176 (1972) 1149-1151. 